\title{
Racionalidad limitada en la valoración de empresas: el caso de Cementos Argos ${ }^{1}$
}

\author{
Andrés David Rodríguez Garzón ${ }^{2}$
}

Recibido: 13 de noviembre del 2018

Aprobado: 6 de diciembre del 2018

Clasificación JEL: D81, G11, G14.

\section{Resumen}

En el artículo se expone una práctica de valoración de empresas realizada a Cementos Argos S. A. En el proceso de valoración se aplicaron diversos métodos, siendo este influenciado por el gran método QCC. Se expone el procedimiento de valoración de la empresa y los cinco tipos de problemas (de la valoración en sí misma, del valorador, del conjunto de información capturada, de las personas constructoras de dicho conjunto de información, y de otros factores) que se tuvieron al valorarla. De esta valoración se concluye que un inversionista actúa bajo racionalidad limitada cuando toma decisiones de inversión en una empresa sobre la que no tiene información completa, pues basa sus decisiones en un conjunto de información cuya confiabilidad es limitada porque tiene ciertas falencias que pueden ocasionar decisiones adversas en el inversionista. La pretensión de este trabajo es ser crítico, eso sí con pruebas empíricas, con algunos dogmas

\footnotetext{
1 Rodríguez, A. D. (2019). Racionalidad limitada en la valoración de empresas: el caso de Cementos Argos. Revista Activos, 16(31), 55-87. DOI: https://doi. org/10.15332/25005278.5316

2 Estudiante de la Facultad de Ciencias Económicas, Universidad Nacional de Colombia, Bogotá D. C. Correo electrónico: anddrodriguezgar@unal.edu.co
} 
de las ciencias económicas, más que exponer hasta el más mínimo detalle de lo que fue la valoración de Cementos Argos.

Palabras clave: conjunto de información, legitimación, racionalidad, valoración de empresas, Cementos Argos, método QCC.

\title{
Limited rationality in the valuation of companies: The Cementos Argos case
}

\begin{abstract}
In the article, a business appraisal practice is exposed to Cementos Argos S.A. In the valuation process, several methods were applied, this being influenced by the great QCC method. The assessment procedure of the company and the five types of problems (of the valuation in itself, of the valuer, of the set of information captured, of the constructors of said set of information, and of other factors) that were discussed when evaluating it. From this assessment it is concluded that an investor acts under limited rationality when making investment decisions in a company over which he does not have complete information, because he bases his decisions on a set of information whose reliability is limited because it has certain shortcomings that can cause adverse decisions in the investor. The aim of this work is to be critical, but with empirical evidence, with some dogmas of the economic sciences, rather than expose even the smallest detail of what was the valuation of Cementos Argos.
\end{abstract}

Keywords: Information set, legitimation, rationality, valuation of companies, Cementos Argos, QCC method. 


\section{Introducción}

Bajo el supuesto de que somos seres humanos racionales, constantemente actuamos con base en "algo" que nos dé confianza para que esa actuación sea lo más racional posible. De esta manera, ese "algo" le da legitimidad a nuestras acciones por ser confiable. Por ejemplo, si queremos tener conocimientos racionales sobre los seres humanos primitivos, simplemente nos basamos en algo confiable, como las profesiones de arqueología y paleoantropología (Comín, 2013), porque consideramos a los respectivos profesionales personas expertas en el asunto. Ahora, piénsese en la moneda: aunque su valor intrínseco es casi nulo, la aceptamos como medio de pago. Según Théret (2015), "la moneda es una institución social, una convención, que se funda en última instancia sobre una fe social, una creencia, una confianza que hace posible su aceptación" (pp.72-73). Él plantea que nuestra aceptación de la moneda como medio de pago se debe a que esta es emitida por una institución soberana: la existencia de tal institución soberana legitima nuestra aceptación de la moneda como medio de pago, pues confiamos en tal institución por el simple hecho de su soberanía.

En ambos ejemplos, los elementos confiables (bancos y profesionales arqueólogos y antropólogos) generan legitimación de nuestras acciones. Hay muchos ejemplos adicionales de cómo nosotros actuamos basados en aspectos confiables. En este documento se muestra tal situación respecto a la conducta de los inversionistas. Estos, como agentes externos de las empresas, al tomar sus decisiones de inversión no cuentan con información completa sobre las empresas, por lo que tienen que hacer uso de la valoración de empresas para poder conocer la realidad económica de estas y tomar sus decisiones de inversión de la manera más racional posible. Como no hay información completa, esa racionalidad es limitada (Hidalgo, 1978). En la valoración de empresas los inversionistas se remiten a un conjunto de información (el elemento "confiable") y con base en este invierten; de esta forma, el conjunto de información legitima sus decisiones de inversión. El conjunto de información se compone de información contable y de información no contable; el inconveniente con este conjunto de 
información es que tiene falencias, lo que hace aún más limitada la racionalidad de las decisiones de los inversionistas porque pueden llegar a tomar decisiones desfavorables. Tal situación es la que se evidencia en la práctica de valoración de empresas realizada a Cementos Argos S. A. como se muestra en el resto del documento.

\section{Metodología}

El documento expone una experiencia de valoración de la empresa Cementos Argos S. A. en Colombia, con el objetivo hipotético de comprarla.

La valoración de empresas tiene propósitos distintos, tales como la transacción parcial o total de una empresa, una fusión, una sinergia, una alianza estratégica, etc. (Parra, 2013). En el caso de la valoración de Argos, se supuso que el propósito era comprarla en su totalidad (toda la participación excluidos los pasivos). Valorar una empresa significa encontrar un rango de valores o importes dentro del cual reside el precio a pagar en la transacción de compraventa de la empresa (Sarmiento y Cayón, 2003). Por eso, aunque en la transacción no está prestablecido el precio a pagar por la empresa, este sí debe encontrarse dentro del rango de valores calculado. De ahí la diferencia entre valor y precio; el precio a pagar está sujeto a diversos factores, tales como la agilidad comercial del vendedor y del comprador.

El rango de valores puede establecerse mediante dos formas:

1. La forma común. Sin partir de ninguna cifra base, se usan distintas técnicas de valoración de empresas, y a partir de todas ellas se establece ese rango.

2. Una cifra base. Se parte de una cifra base ya existente (p. ej. el precio de la acción de la empresa, la cifra de patrimonio contable o la cifra de utilidad contables), y a partir de esta se establecen los límites superior e inferior del rango. Esta es una valoración más rápida; sin embargo, este camino de una cifra base solo es recomendable en unos casos muy específicos y puede ser más arriesgado e inexacto respecto al primer camino (valorar la empresa mediante "la forma común") (Parra, 2013; Santandreu y Torres, 2012). 
Como se muestra en detalle más adelante, en el proceso de valoración de Cementos Argos se intentaron ambas formas: en principio, se valoró la empresa con base en una cifra prestablecida (el precio de la acción); posteriormente, dado que no se encontró fiable la valoración mediante un único indicador, se valoró mediante modelos de valoración más elaborados, como corresponde a "la forma común".

El rango de valores se calculó utilizando todo un conjunto de información disponible sobre la empresa al 12 de mayo de 2017. La valoración de Argos se hizo en cuatro etapas expuestas posteriormente; también se expone una etapa llamada "posvaloración".

Para esta valoración se tomó información contable y no contable. Respecto a la información contable, se tomaron datos de la microcontabilidad y de la macrocontabilidad: respecto a la información microcontable, se tomaron los estados financieros y revelaciones contables de la empresa; respecto a la información macrocontable, se tomaron datos macro obtenidos directa o indirectamente de entidades nacionales como el Dane y el Banco de la República (p. ej. IPP, PIB, precio del petróleo y del dólar, inflación, etc.). La información no contable utilizada fue de dos tipos: información de divulgación propia por parte de la empresa (p. ej. datos generales sobre la misma, reportes integrados, informes de sostenibilidad, acciones en circulación, etc.), e información de la empresa emitida por fuentes ajenas a la empresa (p. ej. precio de la acción, noticias sobre la empresa, reseñas de la empresa, etc.). Todo ese conjunto de información es mostrado en la Figura 1.

Figura 1. Conjunto de información utilizada en la valoración

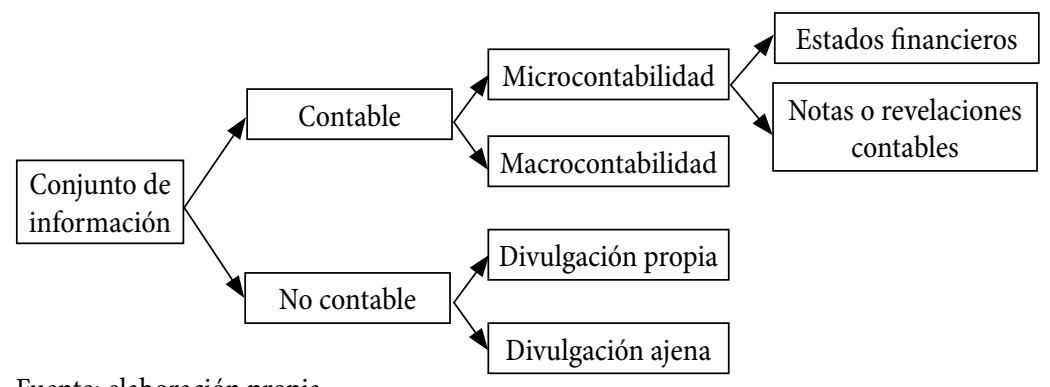

Fuente: elaboración propia. 
En la valoración se utilizaron distintas técnicas o métodos; cada técnica arrojó cifras distintas. El proceso de valoración, incluyendo la elección final del rango de valores, estuvo influenciado por el modelo QCC de valoración de empresas, que se puede consultar en detalle en Santandreu y Torres $(2012)^{3}$.

\section{Proceso de valoración de la empresa}

\section{Etapa 1 de la valoración: diagnóstico general}

En esta etapa no se entró en detalles, pues solo se buscó establecer a partir de un diagnóstico superficial si la empresa era digna de ser comprada. Es necesario este previo diagnóstico superficial para descartar la compra ante la presencia de factores negativos muy obvios (p. ej. escándalos financieros o de otro tipo, quiebra, evidente decadencia financiera, etc.), caso en el cual el diagnóstico evitaría molestias con valoraciones detalladas.

Debido a que se debe conocer el contexto y panorama económico de una empresa para valorarla adecuadamente (Santandreu y Torres, 2012), lo primero que se hizo fue capturar datos sobre dicho panorama, los cuales fueron:

1. Datos básicos de la empresa, como su NIT.

2. Información general de conocimiento común, como los reportes integrados, que mencionaban que la empresa era una fuerte multinacional en el mercado del cemento y que estaba en expansión. Estos reportes también indicaban que su modelo de negocio se basaba en la sostenibilidad ambiental y era "respetuoso de las personas". También se encontró información sobre reconocimientos

3 El modelo QCC no consiste en alguna metodología específica de cálculo, sino en un conjunto de orientaciones a quienes valoren empresas, respecto a tres pilares de la valoración: el qué, el cuándo y el cómo; qué aspectos de la empresa valorar (parte -sus activos- o toda completa -sus activos teniendo en cuenta sus pasivos-), cuándo valorarla (hacia el pasado o hacia el futuro) y cómo valorarla (criterios, variables, conceptos, metodologías, cuantificaciones, etc.). 
internacionales que había recibido la empresa por su sostenibilidad empresarial (desde 2014 la empresa ingresó al Dow Jones Sustainability Index por ser considerada la cementera más sostenible del mundo).

3. Indicadores financieros cruciales de la empresa en los últimos años (2015 y 2016); pero sin entrar en detalles, solo cifras generales como ingresos operacionales, utilidad operacional, utilidad neta, margen neto, EBITDA, etc.

4. La historia de la empresa, que mostraba el hecho de que esta existe desde hace casi un siglo y su paulatina expansión en los siglos XX y XXI por medio de sus adquisiciones.

5. Variables macroeconómicas y políticas coyunturales que afectaban a la empresa como el IPP, el PIB general, el PIB del sector construcción, el precio del petró$\mathrm{leo}^{4}$, el precio del dólar, la inflación y las políticas gubernamentales; también se capturó información sobre la coyuntura laboral y tributaria que atravesaba la economía colombiana en esos momentos e información de tipo internacional. La coyuntura en la valoración de empresas es clave bajo el modelo QCC porque el "cuándo" de la valoración es un pilar clave en el mismo

Ya se contaba entonces, como puede verse, con información de tipo cualitativo y cuantitativo; esta ya empezaba a dar una primera imagen o impresión de Cementos Argos a quien valoraba la empresa:

- al juntar la información coyuntural e histórica de la empresa se tuvo la imagen de una empresa fuerte, sostenible, prometedora y competitiva en el mercado.

- la información de los reportes integrados, los reconocimientos internacionales y sobre todo su inclusión en el índice de Dow Jones generó la imagen de una empresa con comportamientos moralmente aceptables.

4 Los productos de Argos se obtienen de insumos de recursos no renovables derivados del petróleo. Por esta razón el dato del precio del petróleo fue relevante en la valoración la empresa.

5 Pues si se va a pensar en el pasado o en el futuro, debe haber un lapso de tiempo específico como referencia para ello. 


\section{Etapa 2 de la valoración: valoración mediante "una cifra base"}

Desde esta etapa, lo que interesaba de la empresa era más el aspecto cuantitativo que el cualitativo, ya que a partir de los resultados de la etapa 1 se concluyó que Argos era una empresa digna de ser comprada, entonces se prosiguió a determinar si se podía llegar con facilidad al rango de valores mediante el camino de "una cifra base". A partir del modelo QCC se concluyó que las cifras contables nominales de los estados financieros, como la utilidad o el patrimonio, no eran pertinentes para la valoración de Argos, ya que

[...] para cuantificar el valor de una empresa que se encuentra en una situación complicada, con un crecimiento nulo o negativo de las ventas, con dificultades de cobrar de los clientes, perdiendo cuota de mercado y con un equipo de personas desmotivado, serán más relevantes modelos que valoran el pasado, por encima de los que valoran el futuro. Por otro lado, si queremos valorar una empresa que se encuentra en una situación totalmente opuesta a la anterior [...] modelos basados en parámetros futuros, como puede ser el descuento de flujos, serán más adecuados para calcular el valor del proyecto. (Santandreu y Torres, 2012, p. 121).

Argos se encontraba en una situación favorable; por ende, no era adecuado valorarla mediante variables del pasado. Entonces, la cifra base que se probó como mejor indicador del valor fue el precio de la acción en bolsa. Se analizó el precio de la acción ordinaria de Argos en los años 2015 (segunda mitad de año) y 2016, como lo muestra la Figura 2, para evaluar el cumplimiento de la hipótesis de mercado eficiente; pues si el precio de la acción de la empresa ha de ser el mejor indicador de su valor, "el valor bursátil no debe encontrarse sujeto a las distorsiones propias de un mercado de acciones ineficiente" (Parra, 2013, p. 96). En dicha evaluación se encontró como hallazgo una respuesta negativa. 
Figura 2. Precio acción ordinaria Cementos Argos S. A. período julio 2015-diciembre 2016

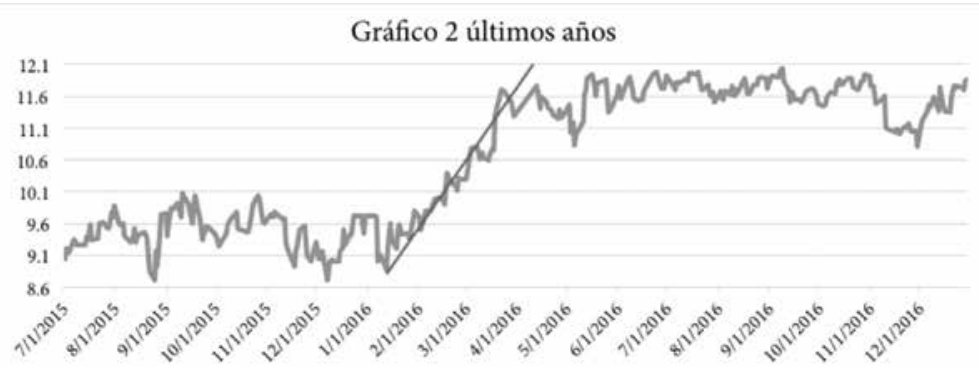

Fuente: elaboración propia en Excel, con datos de la página oficial de Cementos Argos.

$\mathrm{Al}$ analizar el precio de la acción de Argos en el susodicho tiempo se encontró que este había sido relativamente estable: la única variación significativa que tuvo en el tiempo analizado fue en el lapso febrero-marzo del 2016, pues solo hasta inicios de febrero del 2016 empezó a cambiar la tendencia relativamente estable que traía del 2015 para ajustarse (subir) a una nueva tendencia de estabilidad, aproximadamente hacia marzo 17 del 2016. En el primer trimestre del 2016 se emitieron dos noticias relevantes que probablemente causaron la gran subida: la noticia del 29 de enero del 2016 sobre una inversión estratégica de 1000000 de dólares, la cual fue la nueva planta de concreto abierta en el corazón industrial de Medellín; y, la noticia del 25 de febrero del 2016 sobre el incremento del $81 \%$ en las utilidades. Si el mercado fuese eficiente, el 25 de febrero del 2016 el precio de la acción, en respuesta a nueva información relevante, se hubiese ajustado inmediatamente al promedio de $\$ 11600$ que tuvo posteriormente durante el año (2016). Sin embargo, en esos días el precio de la acción estaba en promedio en $\$ 10300$; debía subir y asílo hizo, pero no lo hizo inmediatamente sino que tardó un mes en ajustarse a la nueva tendencia. Debido a que el precio no se ajustó inmediatamente ante nueva información relevante, se concluyó que no se cumplía la hipótesis de mercado eficiente.

Debido a que no se cumplía esta hipótesis, el precio de la acción no era el mejor indicativo del valor de la empresa. Entonces, fue conveniente 
aplicar métodos de valoración más elaborados, en vez del camino de "una cifra base".

\section{Etapa 3 de la valoración: valoración mediante "la forma común"}

En esta etapa se hizo una valoración más detallada y rigurosa. Se debía partir de cero, al concluir que el camino de "una cifra base" no era el adecuado. Fueron aplicados tres tipos de valoración financiera, que son "las tres metodologías genéricas más utilizadas para valorar empresas" (Santandreu y Torres, 2012, p. 117): métodos basados en el balance general, métodos basados en el estado de resultados y métodos basados en flujos de caja.

Valoración basada en el balance general. Aunque el modelo QCC sugiere que para el caso de una empresa prometedora (como lo era Argos en el momento de la valoración) no se deben utilizar métodos de valoración basados en el balance, se utilizaron estos métodos para hacer un contraste posterior con las cifras calculadas bajo los otros métodos. Este contraste es pertinente a modo de verificación. Por ejemplo, cuando ya se sabía que Argos era una empresa con buenas perspectivas, la sugerencia de acuerdo al modelo QCC era que los métodos de valoración más adecuados para la empresa eran aquellos relacionados con flujos de efectivo descontados; por eso, hubiera sido ilógico que los métodos basados en el balance hubiesen arrojado algún valor mayor que los valores calculados bajo los métodos de flujos de efectivo, pues en ese caso el modelo QCC hubiera salido cuestionado. Sin embargo, se utilizaron los tres tipos de métodos (basados en el balance, basados en el estado de resultados y basados en flujos de efectivo) y el resultado de la valoración fue coherente con el planteamiento del modelo QCC, pues la mayor cifra arrojada por los métodos basados en el balance no superó a la menor cifra arrojada por los métodos basados en flujos de efectivo.

En cuanto a los métodos basados en el balance general, se calcularon los siguientes indicadores: 
1. Capital social total.

2. Capital social por acción (capital social total/ acciones en circulación).

3. Valor contable total (el patrimonio total, o sea activo total menos pasivo total).

4. Valor contable por acción ( patrimonio total / acciones en circulación).

5. Valor contable ajustado (el cálculo de este valor es similar al cálculo del valor contable total, pues a los activos se le restan los pasivos, con la diferencia que aquí se ajustan algunos de esos activos y pasivos a su valor respectivo el día exacto de la valoración en vez de tomar los valores a la fecha de cierre (31 de diciembre). Esto, en reconocimiento del hecho de que algunas partidas tal y como aparecen en los estados financieros "no guardan relación con su valor comercial, limitando aún más el resultado (...) para la valoración, para lo cual se toman en cuenta los estudios de valorizaciones, situación que no resuelve la problemática” (Parra, 2013, p. 90). Para la valoración de Argos se hicieron ajustes mediante el uso de variables como la inflación, las tasas de interés de la deuda y precios de mercado).

Para realizar estos cálculos se capturaron datos tales como el capital social, las acciones en circulación, el patrimonio, y en ocasiones se tuvo que ajustar el valor de los activos a valor razonable actual ${ }^{6}$.

En el método de valor contable ajustado, debido a la limitante del tiempo, se calculó el valor razonable de algunos activos de una manera rudimentaria (simplemente se tomaron las cifras del 31 de diciembre y se les imputó una inflación), y para otros activos cuya medición era compleja (p. ej. instrumentos financieros derivados, inversiones en subsidiarias, etc. ) se supuso que su valor razonable se había mantenido constante desde diciembre (un supuesto muy fuerte) debido a que no se tenía información para calcular el valor razonable (no se conseguía en los estados financieros o en otro medio) o se tenía algún tipo de información pero no era muy claro cómo poder usarla para calcular el valor razonable de los activos. Ahora, si

6 O sea, los estados financieros proporcionaban el valor razonable de los activos (los que se miden a valor razonable) al 31 de diciembre del 2016, pero la valoración se hizo al 12 de mayo del 2017; sin embargo, para el método de valor contable ajustado se debe actualizar el valor razonable de activos y pasivos a la fecha de valoración. 
la compra hubiese sido real y no solo una práctica académica de valoración, el inversionista valorador también hubiera tenido el problema temporal. Si una empresa se quiere valorar con mayor minuciosidad y rigurosidad hay que dedicarle más tiempo; sin embargo, es necesario sacrificar algo de minuciosidad y rigurosidad para poder tomar una decisión oportuna y actuar rápido en la transacción de la empresa, y más aún en estos casos en donde la empresa a comprar es prestigiosa y prometedora.

Otro indicador de valoración de empresas correspondiente a los métodos basados en el balance general es el valor neto de liquidación. Este consiste en tomar el valor del patrimonio y restarle los costos de liquidación de la empresa; ${ }^{7}$ el indicador estima el valor que tendría la empresa "ante una eventual liquidación de la misma” (Parra, 2013, p. 91). Por eso, este indicador es el método de valoración ideal para empresas que se encuentren en proceso de liquidación. La razón por la que se ignoró este indicador en la valoración de Argos fue que los resultados de la etapa 1 indicaron que la empresa tenía excelentes indicadores y proyecciones a futuro, por lo que claramente se encontraba bajo la hipótesis de un negocio en marcha y no en proceso de liquidación. Aquí se puede evidenciar cómo el conjunto de la información contable y no contable, al otorgar confiabilidad, puede incluso legitimar el que en la valoración se opte por uno o por otro método.

Valoración basada en el estado de resultados. En cuanto a los métodos basados en el estado de resultados, se calcularon los siguientes cuatro indicadores:

Valoración por PER

Valor de la empresa por PER $=$ PER $2017(\mathrm{~A})^{\star}$ Utilidad esperada 2017 (B)

(A) PER 2017 $=\frac{\text { Precio de mercado acción } 2017}{\text { UPA } 2016}$

7 Tales como costos de desmantelamiento de activos fijos, pagos de indemnizaciones y honorarios del liquidador. 
De donde, PER 2016= $\frac{\text { utilidad } 2016}{\text { acciones en circulacion }}$

(B) UTIL. ESPERADA $2017=$ UTILIDAD 2016* $(1+$ inflación proyectada 2017)

Se aplicó la valoración por múltiplos a partir de tres variables distintas del estado de resultados: las ventas, el EBITDA y las utilidades. Sin embargo, a estos indicadores se les dio una importancia menor por estar basados en cifras estáticas y tener mayor subjetividad.

El múltiplo se calculó así:

$$
\text { Múltiplo }=\text { PER } 2017 \times \frac{U O D I^{*}}{U P A 2016}
$$

* En la Tabla 1 se puede apreciar cómo se llega a esta cifra.

Con este múltiplo se calcularon tres indicadores:

1. Múltiplo de las ventas $=$ Ventas netas $2016 \times$ Múltiplo

2. Múltiplo del EBITDA $=E B I T D A^{* *} \times$ Múltiplo

** En la Tabla 1 se puede apreciar cómo se llega a esta cifra.

3. Múltiplo de las utilidades $=U N V H$ Promedio ${ }^{* * *} \times$ Múltiplo

*** Promedio de las utilidades netas de los últimos 7 años a valor de hoy ${ }^{8}$.

8 Las utilidades netas desde el año 2011 y hasta el 2017 se convirtieron a valor del 2017, y se promediaron los 7 resultados. Para la conversión de las 7 utilidades netas se usó la fórmula en Excel =VF(tasa; nper;;[va]); donde la "tasa" fue la inflación de cada año (obviamente, para 2017 se utilizó la inflación proyectada), "nper" el período transcurrido para cada año hasta 2017, y "[va]" la utilidad neta del año respectivo (para 2017 se utilizó la utilidad neta proyectada (utilidad neta $2016^{\star}(1+$ inflación proyectada 2017))). Así, por ejemplo, el "nper" para el cálculo del valor de la utilidad neta del 2011 a valor de 2017 fue 6, el "nper" para el cálculo del valor de la utilidad neta del 2012 a valor del 2017 fue 5, y el "nper" para el cálculo del valor de la utilidad neta del 2017 a valor del 2017 fue, obviamente, 0 ; de hecho, no era necesario convertir la utilidad neta del 2017 porque obviamente ya estaba a su "valor de hoy". 
En la valoración por PER, para la imputación del precio de mercado, se supuso que lo correcto era tomar el precio promedio de las cotizaciones diarias de lo corrido del año 2017, debido a que, en concepto del valorador, el precio de la acción era estable en ese período (enero-mayo) porque la dispersión y diferencia (rango) entre el mínimo y el máximo de los precios no eran muy grandes. Pero, ¿por qué no se usó otro precio? ¿Por qué no usar el precio del día de valoración? ¿Por qué no el precio más bajo de la serie enero-mayo? Estas preguntas muestran que la valoración de empresas no está libre de la subjetividad de los valoradores; el conjunto de herramientas utilizado para la valoración está afectado "por el riesgo y por aspectos subjetivos, lo que lleva a resultados diversos que dependen inclusive de quién hace la valoración" (Parra, 2013, pp. 89-90).

Respecto a la inflación proyectada en dos de los métodos, se tuvo el inconveniente de que era más de una fuente la que arrojaba datos sobre la inflación: el Dane, economistas, analistas, académicos, Bancolombia, Banco de la República, Fedesarrollo, etc. Estas fuentes tenían opiniones encontradas sobre el dato de la inflación de los años venideros, después de aprobada la reforma tributaria 2016. Surgieron dilemas sobre la cifra de inflación a utilizar en la valoración, como el siguiente: ¿se debe tomar el dato de la inflación de la fuente que se considere más confiable, o se debe tomar una cifra promedio de tales fuentes? Aquí también se puede evidenciar que, óptese por lo que se opte, siempre estará involucrada la subjetividad de quien valora.

Valoración basada en flujos de efectivo. Aquí se calcularon los flujos de caja libre (FCL) proyectados, como se muestra en la Tabla 1, para los años 2017, 2018 y 2019. Como lo sugiere el método QCC, mediante los FCL se llega al valor que se pagaría al comprar la empresa sin pasivos; por eso se usaron FCL y no FCA (flujos de caja del accionista), y posteriormente se utilizó el costo promedio ponderado de capital (CPPC) para descontar esos flujos en vez de solamente el costo del patrimonio. Para calcular esos FCL proyectados se tuvieron que elaborar estados financieros proyectados; elaboración donde también se presentaron supuestos e inconvenientes. 
Tabla 1. Procedimiento de cálculo del flujo de caja libre (FCL) aplicado en la valoración

\begin{tabular}{l|c}
\hline Ventas netas & $\mathrm{XXX}$ \\
\hline Costos operacionales en efectivo & $(\mathrm{XXX})$ \\
\hline Otros ingresos operacionales & $\mathrm{XXX}$ \\
\hline EBITDA & $\mathrm{XXX}$ \\
\hline Costos operacionales sin desembolso & $(\mathrm{XXX})$ \\
\hline UTILIDAD OPERACIONAL (UAII) & $\mathrm{XXX}$ \\
\hline Impuesto aplicado 33 \% & $(\mathrm{XXX})$ \\
\hline NOPAT (UODI) & $\mathrm{XXX}$ \\
\hline Costos operacionales sin desembolso & $\mathrm{XXX}$ \\
\hline FLUJO DE CAJA BRUTO operacional & $\mathrm{XXX}$ \\
\hline Disminución del KTNO & $\mathrm{XXX}$ \\
\hline Compra propiedad, planta y equipo & $(\mathrm{XXX})$ \\
\hline Compra propiedad de inversión & $(\mathrm{XXX})$ \\
\hline venta negativa propiedad, planta y equipo y propiedad de inversión & $(\mathrm{XXX})$ \\
\hline Adquisición de inversiones en asociadas-negocios conjuntos & $(\mathrm{XXX})$ \\
\hline Venta de instrumentos financieros & $\mathrm{XXX}$ \\
\hline Compra de intangibles & $(\mathrm{XXX})$ \\
\hline Dividendos recibidos (de inversiones en otras empresas) & $\mathrm{XXX}$ \\
\hline Otros flujos de efectivo actividades de inversión & $\mathrm{XXX}$ \\
\hline FLUJO DE CAJA LIBRE & $\mathrm{XXX}$ \\
\hline Pago servicio de la deuda & $(\mathrm{XXX})$ \\
\hline Pago de pasivos arrendamiento financiero & $(\mathrm{XXX})$ \\
\hline Adquisición de nuevos prestamos & $\mathrm{XXX}$ \\
\hline Pago de bonos en circulación & $(\mathrm{XXX})$ \\
\hline FLUJO DE CAJA ACCIONISTAS & $\mathrm{XXX}$ \\
\hline
\end{tabular}

Fuente: elaboración propia en Excel.

Se tuvieron varios problemas en el cálculo de las cifras proyectadas para llegar a los FCL.

De antemano, proyectar el futuro es complicado. No se puede saber con certeza qué factores imprevisibles se pueden presentar, tanto a nivel 
general como a nivel de la empresa, que alteren de forma significativa las cifras de las proyecciones: no se sabe cómo evolucionarán las inversiones estratégicas de la empresa o el Gobierno de empresa; no se sabe si ocurrirá una gran catástrofe natural o una gran crisis económica. Para el caso puntual de Argos, lo que ocurra en el mercado de valores es especialmente importante para la empresa, pero no se puede saber con certeza sobre los futuros acontecimientos que ocurran en este; por ejemplo, Bezemer (2012) y Santandreu y Torres (2012) sacan a colación el hecho de que muy pocos analistas y predictores acertaron sobre la gran crisis de 2008, pues casi ninguno la vio venir.

Se proyectaron todas las cifras de los estados financieros de Argos. A unas partidas le fueron modificadas sus cifras al hacer las proyecciones, por considerarse partidas críticas o cruciales para la empresa (las ventas, las cuentas por cobrar, el movimiento de la propiedad planta y equipo, pasivos comerciales, pasivos por impuestos, pasivos laborales, cargas prestacionales, capital social, deuda financiera, inventarios y costo de ventas). A muchas otras partidas le fueron dejadas estáticas ${ }^{9}$ sus cifras en las proyecciones, bien por considerarse irrelevantes por cuantía (gastos pagados por anticipado, provisiones, ingresos recibidos por anticipado, otros pasivos, pasivos por impuestos diferidos, acciones propias readquiridas, pérdida por medición en obligaciones de beneficios definidos, otros ingresos operacionales netos), o bien porque se consideró que había un gran margen de incertidumbre al ajustarlas (inversiones en subsidiarias, asociadas y negocios conjuntos, instrumentos financieros derivados, activos intangibles, activos biológicos, ganancias no realizadas, deterioro del valor, ingresos financieros, diferencia en cambio, participación en el resultado de asociadas y negocios conjuntos).

En cuanto a las partidas que se modificaron en las proyecciones, el problema fue hacer estimaciones precisas. Las estimaciones precisas ya se sabe

9 O sea, que se les imputó para los años proyectados posteriores, el mismo importe que tenían para el año 2016. 
que no son gratuitas, por lo que se tiene que recurrir a estimaciones que arrojen un valor aproximado, tal como se hizo con Argos; téngase en cuenta, por ejemplo, que un inversionista valorador difícilmente emprenderá una búsqueda de activos de la empresa para realizarles peritaje.

No solo se tuvieron problemas con las partidas presentadas en los estados financieros; también con las revelaciones contables. Por ejemplo, en algún punto fue necesario estimar la carga prestacional, pero la revelación de los gastos de personal era muy restringida para ello; de todos modos, el valorador "tuvo que arreglárselas" y hacer supuestos para estimar la carga prestacional a partir de ese único dato. También fue necesario tener el costo de ventas por separado para los productos cemento y concreto, pero se presentaba una sola cifra de costo de ventas. Estos ejemplos evidencian que las revelaciones contables y la forma como se presentan (por ejemplo, su nivel de agregación y detalle) inciden en la valoración de empresas.

No solo se hicieron supuestos en la construcción de los estados financieros proyectados, sino también en los FCL proyectados a partir de tales estados; por ejemplo, el valorador aplicó ciertos criterios para determinar cuáles activos y cuáles pasivos corrientes operativos debían hacer parte del cálculo del capital de trabajo neto operativo (KTNO).

Se construyeron los estados financieros proyectados para los años 2017, 2018 y 2019; a partir de estos, se obtuvieron para los mismos años respectivos los FCL proyectados usando el procedimiento de la Tabla 1. Ahora, había que expresar esos FCL proyectados a valor presente (a valor de 2017). Surgió otro inconveniente: ¿qué tasa de descuento utilizar para cada uno de esos FCL proyectados? Aunque hay varios puntos de vista sobre esta cuestión (p. ej. utilizar una simple inflación, una simple tasa de algún activo financiero, etc.), en la valoración se utilizó el CPPC, como lo sugiere el modelo QCC.

El inconveniente es que el CPPC de cada uno de los años se calculó, obviamente, a partir de algunas de las cifras proyectadas, y como se vio, en la construcción de las mismas se hicieron importantes juicios de valor; 
estos juicios pueden causar variaciones respecto a los CPPC reales ${ }^{10}$, pero "variaciones que a primera vista pueden parecer poco importantes de cualquiera de las variables en un descuento de flujos, pueden llevar a fluctuaciones muy relevantes en el valor final" (Santandreu y Torres, 2012, p. 123). Como se sabe, para calcular el CPPC es necesario calcular el costo de patrimonio. Este cálculo tampoco estuvo libre de subjetividades y supuestos.

La fórmula para calcular el costo de patrimonio es:

$$
K e=R f+[(R m-R f) \star \beta]
$$

Donde, $\mathrm{Ke}=$ costo de patrimonio, $\mathrm{Rm}=$ rendimiento de mercado, $\mathrm{Rf}=$ tasa libre de riesgo ${ }^{11}$

Para el cálculo del beta $(\beta)$, se utilizó la cifra del beta de Damodaran ${ }^{12}$ para el sector de la construcción; a esta cifra se le añadieron unos cuantos puntos adicionales para incluir el efecto del "riesgo país". Para calcular la tasa libre de riesgo se buscaron tasas de distintos TES del Estado y se eligió la que se consideraba más adecuada. Para calcular el rendimiento del mercado $(\mathrm{Rm})$, se realizó el siguiente procedimiento:

1. Se tomaron todas las 246 cifras del Colcap (un índice del mercado de valores colombiano) del último año; es decir, se tomaron 246 datos desde la fecha $\mathrm{A}_{1}$ hasta la fecha $\mathrm{A}_{246}$, siendo $\mathrm{A}_{1}$ el 13/05/2016 y $\mathrm{A}_{246}$ el 12/05/2017 $7^{13}$. Las 246 cifras simbolizadas desde $\mathrm{B}_{1}$ hasta $\mathrm{B}_{246 \text {. }}$

2. Con base en dichas cifras, se construyó una tabla de escenarios de comportamiento de mercado para hallar el valor esperado del rendimiento diario

10 Obviamente desconocidos en el momento de la valoración de la empresa.

11 Una explicación detallada de estos conceptos para quien no los maneje se puede consultar en Santandreu y Torres (2012).

12 Los betas por actividad económica pueden consultarse en http://pages.stern. nyu.edu/ adamodar/New_Home_Page/datafile/Betas.html

13 Los datos fueron 246 y no 365 debido a la dinámica del mercado. 
del mercado (Rm diario esperado). La tabla construida fue la Tabla 2. Para construirla, se establecieron cinco intervalos, asociados a cinco escenarios de rendimiento o comportamiento del mercado (muy malo, malo, regular, bueno y muy bueno).

Tabla 2. Escenarios de comportamiento de mercado

\begin{tabular}{|c|c|c|c|c|c|}
\hline \multirow[b]{2}{*}{$\begin{array}{c}\text { Escenarios de } \\
\text { comportamiento }\end{array}$} & \multicolumn{2}{|c|}{ Intervalo } & \multirow[b]{2}{*}{$\mathbf{P}$} & \multirow[b]{2}{*}{ MI } & \multirow[b]{2}{*}{$\mathbf{P}^{\star} \mathbf{M I}$} \\
\hline & $\begin{array}{l}\text { Valor del } \\
\text { límite } \\
\text { inferior }\end{array}$ & $\begin{array}{l}\text { Valor del } \\
\text { límite } \\
\text { superior }\end{array}$ & & & \\
\hline Muy malo & Bmin & $\begin{array}{l}\text { C (Bmin } \\
+ \text { Long })\end{array}$ & $\begin{array}{c}\mathrm{P} 1 \\
(\mathrm{H} 1 / 246)\end{array}$ & $\begin{array}{c}\mathrm{MI1} \\
([\mathrm{Bmin}+\mathrm{C}] / 2)\end{array}$ & $\mathrm{P} 1^{\star} \mathrm{MI} 1$ \\
\hline Malo & $\mathrm{C}$ & $\begin{array}{l}\mathrm{D}(\mathrm{C}+ \\
\text { Long) }\end{array}$ & $\begin{array}{c}\text { P2 } \\
(\mathrm{H} 2 / 246)\end{array}$ & $\begin{array}{c}\text { MI2 } \\
([\mathrm{C}+\mathrm{D}] / 2)\end{array}$ & $\mathrm{P} 2{ }^{\star} \mathrm{MI} 2$ \\
\hline Regular & $\mathrm{D}$ & $\begin{array}{l}\mathrm{E}(\mathrm{D}+ \\
\text { Long) }\end{array}$ & $\begin{array}{c}\text { P3 } \\
(\mathrm{H} 3 / 246)\end{array}$ & $\begin{array}{c}\mathrm{MI} 3([\mathrm{D}+\mathrm{E}] \\
) / 2)\end{array}$ & $\mathrm{P} 3{ }^{\star} \mathrm{MI} 3$ \\
\hline Bueno & $\mathrm{E}$ & $\begin{array}{l}\mathrm{F}(\mathrm{E}+ \\
\text { Long) }\end{array}$ & $\begin{array}{c}\mathrm{P} 4 \\
(\mathrm{H} 4 / 246)\end{array}$ & MI4 ([E+F]/2 & $\mathrm{P} 4^{\star} \mathrm{MI} 4$ \\
\hline \multirow[t]{2}{*}{ Muy bueno } & $\mathrm{F}$ & $B \max$ & $\begin{array}{l}\mathrm{P} 5(\mathrm{H} 5 \\
/ 246)\end{array}$ & $\begin{array}{c}\text { MI5 } \\
([\mathrm{F}+\mathrm{Bmax}] / 2)\end{array}$ & $\mathrm{P}^{\star} \mathrm{MI} 5$ \\
\hline & & & & Suma & $\begin{array}{c}\text { Rm diario } \\
\text { esperado }\end{array}$ \\
\hline
\end{tabular}

Fuente: elaboración propia.

Los límites de cada intervalo se fijaron utilizando los valores Bmin, Bmax y Long. Siendo Bmin el menor valor desde B1 hasta B246, o sea el menor valor de las cifras Colcap tomadas; siendo Bmax el mayor valor desde B1 hasta B246; y, siendo Long la longitud de cada intervalo, obtenida así:

Long $=$ Rango $/ 5$; donde Rango $=\mathrm{Bmax}-\mathrm{Bmin}$.

Adicionalmente, para cada intervalo se calcularon las probabilidades (P) de ocurrencia y las medias de intervalo (MI). La probabilidad para cada intervalo es la probabilidad de que el mercado se comporte en el futuro de acuerdo al respectivo escenario, y para el caso de Argos fue definida de acuerdo a la 
observación de los 246 datos tomados del Colcap ${ }^{14}$; no sobra decir que la suma de las $\mathrm{H}(\mathrm{H} 1, \mathrm{H} 2, \mathrm{H} 3, \mathrm{H} 4, \mathrm{H} 5)$ fue igual a 246 como debía ser.

4. Con las probabilidades (P) y las medias de intervalo (MI) se llegó al valor esperado del rendimiento diario del mercado ( $\mathrm{Rm}$ diario esperado), como se puede apreciar en la Tabla 2.

5. Como se tenía un Rm diario y el Rm de la fórmula del costo de patrimonio es anual, se convirtió el Rm diario utilizando la tasa de interés efectiva ${ }^{15}$.

En este cálculo del costo de patrimonio (Ke) también se presentó subjetividad por parte del valorador: al imputar el riesgo país, al seleccionar activos financieros (TES) específicos, al seleccionar la muestra del listado de índices Colcap y al elegir el número de intervalos en la Tabla 2, entre otras situaciones.

Con este costo de patrimonio se procedió a calcular los costos de capital (CPPC) proyectados para los años 2017, 2018 y $2019^{16}$. Al tener proyectados tanto los FCL como los CPPC para los distintos años, se calcularon los valores presentes de los FCL de tales años (VPFCL2017, VPFCL2018 y VPFCL2019) tomando como tasa de descuento a los CPPC. A partir de estos VPFCL se procedió a calcular tres indicadores correspondientes a tres distintos valores de la empresa: VEFCL1, VEFCL2 y VEFCL3; VEFCL es valor de la empresa a partir de los flujos de caja libre. VEFCL1 tuvo en cuenta tanto la inflación como la constante g de crecimiento a perpetuidad; VEFCL2 solo tuvo en cuenta la inflación; y, VEFCL3 no tuvo en cuenta ni siquiera la inflación. Por eso, al incorporar distintos supuestos se obtuvieron tres distintos valores de la empresa a partir de los flujos de efectivo, donde naturalmente:

\section{VEFCL1 > VEFCL2 >VEFCL3}

14 Por ejemplo, para la probabilidad del intervalo "muy malo" (P1), H1 fue 3.3 fue el número de datos de los 246 del año en los que el índice Colcap correspondió al rendimiento "muy malo", o en otras palabras, el número de veces de las 246 en las que el índice del Colcap se ubicó entre los valores Bmin y C.

15 Esta conversión, en Excel, se hizo así: =INT.EFECTIVO (G17^365; 365); siendo G17 la celda donde se hallaba la cifra del Rm diario esperado.

16 Se supuso un mismo costo de patrimonio para los años 2017, 2018 y 2019. 


\section{Etapa 4 de la valoración: establecimiento del rango de valores}

Ya calculadas todas las cifras (doce en total) bajo los tres distintos tipos de valoración (basados en el balance, basados en el estado de resultados y basados en flujos de efectivo), se procedió a establecer el rango de valores de la empresa.

Los doce valores calculados por los tres distintos tipos de valoración fueron ordenados de mayor a menor, así:

\section{VEFCL1}

2. Valor de la empresa por PER

3. VEFCL2

4. VEFCL3

5. Valor contable total

6. Valor contable ajustado

7. Capital social total

8. Múltiplo de las ventas

9. Múltiplo de las utilidades

10. Múltiplo del EBITDA

11. Valor contable por acción

12. Capital social por acción

Los valores 11 y 12 obviamente fueron los más bajos; estos se calcularon solamente para tener la noción del valor individual de la acción, pero debían ser inmediatamente descartados en el establecimiento del rango de valores ya que lo que se quería era comprar toda la empresa y no algún grado de participación específica.

Como se comentó antes, el modelo QCC sugirió que los métodos más apropiados para establecer el valor de Argos, dado que la empresa tenía muy 
buenas perspectivas a futuro, eran aquellos basados en flujos de efectivo y no aquellos basados en el balance. Ya que ninguno de los valores calculados mediante los métodos basados en el balance superó al menor valor de los valores calculados mediante flujos de efectivo, debían descartarse los valores desde el 5 hasta el 10.

Quedaron entonces los valores del 1 al 4 para establecer el rango de valores. El rango de valores perfectamente pudo ser VEFCL3 ----- VEFCL1, pero se prefirió tomar al indicador PER como límite inferior del rango de valores. Se estableció el rango final de valores de negociación en Valor PER ----- VEFCL1, dentro del cual Cementos Argos podría ser transada en su totalidad el 12 de mayo de 2017; en otras palabras, el inversionista valorador de la empresa (en posición de comprador) esperaría tener que pagar como mínimo por Argos el Valor PER y estaría dispuesto a pagar como máximo el valor VEFCL1.

Con esto último terminó el proceso de valoración de Cementos Argos. Se tuvieron muchos más problemas y supuestos específicos en la valoración, pero en este documento se mencionaron solamente los más generales y críticos.

La información capturada en la valoración de Argos, tanto cualitativa como cuantitativa, fue preparada por distintas fuentes: contadores, gerentes, revisores fiscales, la BVC (Bolsa de Valores de Colombia), el Dane, bancos, académicos, periodistas, analistas y el Gobierno, entre otras personas e instituciones. Estas fuentes proporcionan cierto nivel de confianza, por lo que legitiman las decisiones de los valoradores de empresas. En la valoración de Argos se evidenció la legitimación causada por dos distintos grupos de fuentes: fuentes ajenas a la empresa y fuentes de la empresa.

En cuanto a las fuentes ajenas a la empresa, estas emiten pronunciamientos cualitativos y cuantitativos sobre ella y de este modo generan cierto comportamiento en el inversionista valorador; sin embargo, no necesariamente esos pronunciamientos están libres de sesgo o error, cuestión de la 
cual la historia puede dar cuenta ${ }^{17}$. Incluso, las fuentes ajenas a la empresa pueden incidir en la información emitida por las fuentes de la empresa; por ejemplo, la información contable de esta puede ser afectada por instituciones externas a la misma. Considérese que en el modelo contable de las NIIF se pueden tener dos tipos de representaciones financieras según el criterio de poder adquisitivo: basadas en importes nominales o basadas en importes reales (ajustadas a la inflación). La NIC 29 determina que:

En una economía hiperinflacionaria, la información sobre los resultados de las operaciones y la situación financiera en la moneda local sin reexpresar no es útil. La moneda pierde poder de compra a tal ritmo que resulta equívoca cualquier comparación entre las cifras procedentes de transacciones y otros acontecimientos ocurridos en diferentes momentos del tiempo, incluso dentro de un mismo período contable. (Párr. 2).

La NIC 29, aunque deja al criterio profesional la decisión de cuándo se deben llevar estados financieros acordes con una economía hiperinflacionaria, presenta unos indicios de cuándo se presentan situaciones de hiperinflación; de estos indicios, el más fácil y trivial de aplicar para los preparadores de información financiera es el criterio de que "la tasa acumulada de inflación en tres años se aproxima o sobrepasa el 100 \%"(IASB, 2016, párr. 3). En Colombia es principalmente el Dane quien determina el dato de inflación, una entidad pública de orden nacional que a primera vista parece confiable por ser parte de la estructura estatal. Si el Dane emitiera datos que mostraran hiperinflación, se tendría que llevar contabilidad hiperinflacionaria y por ende ajustar la información financiera. Más importante que pensar en la diferencia matemática entre una contabilidad nominal y una ajustada a la inflación, es pensar en cómo una entidad como el Dane es quien marca la diferencia entre llevar una contabilidad a valores nominales y una a valores reexpresados; pues los preparadores de información financiera, con base en los datos de inflación, determinan bajo qué modelo

17 Por ejemplo, en la crisis subprime, las agencias calificadoras de riesgo daban a varias inversiones una errónea calificación AAA y de esta forma influían en las decisiones adversas de los inversionistas. 
contable (nominal o real) preparar la información financiera. Por ello, los datos macro legitiman decisiones de los valoradores de empresas ya sea directamente, o vía microcontabilidad (como se puede ver con el tema de la inflación en los estados financieros).

En la valoración de Argos el dato de la inflación fue utilizado en varias ocasiones: como tasa de descuento, para calcular la apreciación de algunos activos, para calcular las ventas proyectadas, etc. Para el valorador fue legítimo el dato de la inflación por el hecho de provenir de una entidad estatal reconocida (el Dane). Esta situación es análoga al planteamiento de Théret (2015) para el caso de los bancos y la aceptación de la moneda. Sin embargo, también se encontraron otros datos de inflación tanto histórica como esperada, arrojados por otras fuentes, que hubiesen podido ser legitimados. Por eso, se presentó el dilema de a cuál de las fuentes creerle; la elección implicó seguir unos supuestos que incidieron en la valoración de la empresa. Además de la inflación, otros datos fueron legitimados en la valoración. Se legitimaron datos de instituciones que bien podrían no ser lo suficientemente independientes en la emisión de sus datos (como el Dane y el Banco de la República). La independencia de criterio de quienes emiten la información a ser usada por agentes económicos para tomar decisiones es un tema crítico (Stiglitz, 2003), puesto que los datos emitidos por ellos inciden en últimas en la decisión de los valoradores de empresas.

Respecto a las fuentes de la empresa, cabe preguntarse si realmente son idóneas esas fuentes de información. Por ejemplo, los preparadores de información financiera pueden no ser lo suficientemente idóneos debido a deficiencias en su preparación profesional, su responsabilidad y su ética. ¿Cómo puede un valorador de empresas saber sobre esto? ¿Qué le garantiza que los valores de los informes financieros estén preparados legalmente y éticamente? ¿Cómo puede llegar a determinar en un tiempo razonable y oportuno si los informes de responsabilidad social tienen fundamento o son declaraciones caprichosas?

Además, hay que tener en cuenta que la información capturada en el proceso de valoración puede tener falencias ocasionadas no por la voluntad 
de las personas involucradas con dicha información, sino por cuestión de metodología; pues una determinada metodología con la que la información es construida puede afectar las decisiones de los valoradores de empresas. Por ejemplo, la contabilidad se puede preparar legalmente, pero eso no garantiza que esta se corresponda con la realidad económica de la empresa. Las NIIF son una forma específica de llevar contabilidad respecto a otros tipos de modelos contables existentes; el hecho de que la contabilidad de Cementos Argos estuviera preparada bajo NIIF y no bajo otro modelo contable, incidió en la valoración de la empresa. Por otro lado, en la etapa 1 de diagnóstico inicial, se averiguó por el desempleo. El que el Dane use una u otra metodología al calcular el desempleo incide en la imagen percibida por los valoradores de empresas al analizar el sector económico de la empresa que están valorando.

Todas las anteriores situaciones comentadas para los dos distintos grupos de fuentes, evidencian las falencias que puede presentar el conjunto de información. Todas estas falencias hacen que la racionalidad de las decisiones del inversionista en la valoración de empresas sea limitada; es limitada en el sentido de que cabe la posibilidad de que el inversionista tome una decisión adversa. Tal situación pudo experimentarse con Cementos Argos después de las cuatro etapas de valoración.

\section{Etapa posvaloración}

El 13 de octubre de 2017, meses después de la valoración de Cementos Argos, se hizo pública la noticia de que la empresa probablemente había participado en un cartel del cemento en compañía de Cemex y Holcim. De acuerdo con una investigación de la Superintendencia de Industria y Comercio:

Estas tres empresas se pusieron de acuerdo para cometer las conductas de [...] fijación de precios del cemento entre el año 2010 y 2013 provocando un alza de hasta un $30 \%$ en el valor del concreto y [...] repartición del mercado por medio de la cual se establecieron zonas exclusivas para la comercialización del mismo. (El Tiempo, 2017). 
Hasta ese momento la participación de Argos en el cartel era solamente un ambiguo rumor; pero si dicha participación llegaba a confirmarse, sin duda se tendría como resultado una conducta inmoral por parte de la empresa, pues:

La cartelización es una conducta que atenta contra el bienestar general de los consumidores, el buen funcionamiento de la economía de mercado y en la mayoría de los casos de manera más grave a la población más pobre. Cuando hay carteles de precios los productos se vuelven inalcanzables para los más pobres. (Robledo, 2017).

Después de hacerse público el rumor sobre el cartel del cemento, durante el mes y medio siguiente el precio de la acción ordinaria de Argos se desplomó en $\$ 1240$, pues el 29 de noviembre de 2017 tuvo un precio de $\$ 10$ 540, el más bajo en todo el año (ver Figuras 3 y 4). Obsérvese en la Figura 3 que el día antes de la noticia (12 de octubre de 2017) el precio de la acción era de $\$ 11780$. Compárese en tal figura el antes y el después de tal fecha: se puede observar que en los cuatro meses anteriores a tal fecha el precio de la acción tenía una tendencia estable; ahora, obsérvese que después de la fecha de la noticia la tendencia estable se altera y va a la baja: prácticamente todos los precios del mes posterior fueron inferiores a los que se venían presentando antes de la emisión de dicha noticia ${ }^{18}$.

El 12 de diciembre de 2017, dos meses después del rumor, la conducta inmoral dejó de ser rumor y fue corroborada:

Por cartelización empresarial para la fijación de los precios ex fábrica del cemento gris Portland Tipo 1 en el mercado nacional, la Superintendencia de Industria y Comercio impone sanciones a ARGOS, CEMEX, HOLCIM y 6 altos directivos de estas empresas por un valor global superior a \$200 MIL millones de pesos... la sanción tiene como causa, la existencia de un acuerdo en la modalidad de

18 Si se piensa esta situación a la luz de la hipótesis de mercado eficiente, puede plantearse que esta sí se cumple para Argos ante la divulgación de malas noticias, pero no ante la divulgación de buenas noticias, como se vio antes. 
Figura 3. Caída del precio de la acción ordinaria de Cementos Argos después de la noticia del 13 de octubre

- cemargos - pFCEMARGos

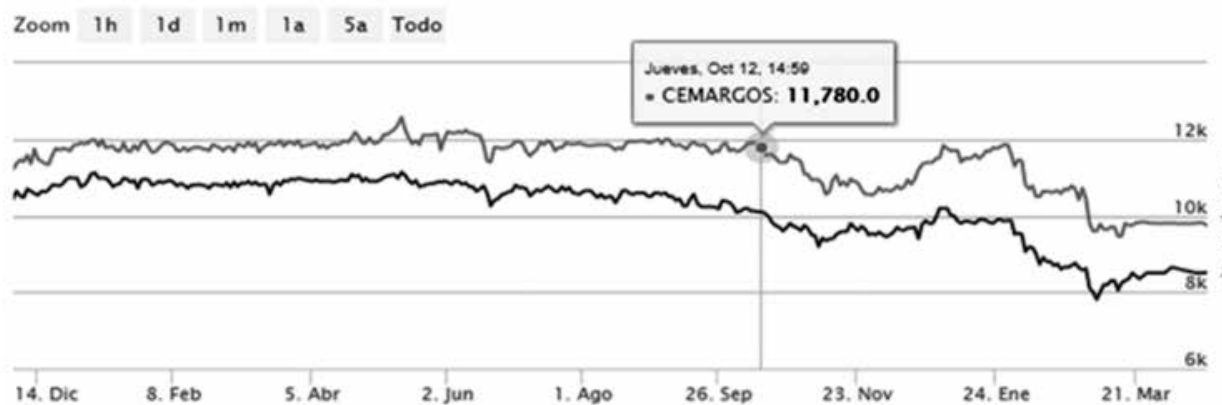

Fuente: página oficial de la empresa. https://www.argos.co/ir/informacion-financiera/accion

Figura 4. Precio de la acción el 29 de noviembre de 2017

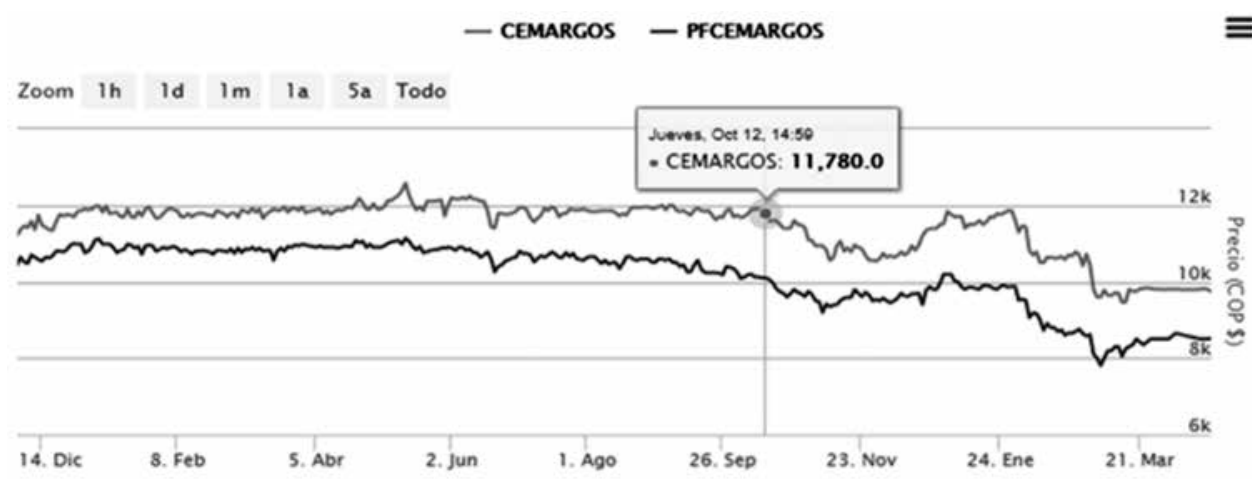

Fuente: página oficial de la empresa. https://www.argos.co/ir/informacion-financiera/accion

paralelismo consciente para la fijación de los precios... durante el período objeto de la investigación, es decir, entre enero de 2010 y diciembre de 2012 (3 años)... ARGOS, CEMEX y HOLCIM, durante el período investigado, mantuvieron un ambiente colaborativo, no competitivo, de colegaje o de amiguismo empresarial extraño a un mercado en competencia, que deja en evidencia que se abstuvieron, de manera consciente y coordinada, de 
competir efectivamente, tal y como se advierte en diferentes documentos, correos electrónicos, comunicaciones internas, entre otras evidencias materiales probatorias. (Superintendencia de Industria y Comercio, 2017).

En esta segunda ocasión no se puede notar una caída inmediata del precio de la acción como consecuencia de la confirmación de la mala conducta de Argos. Sin embargo, posteriormente se puede observar que en los primeros meses del 2018 la acción se desploma aún más; por ejemplo, el 13 de marzo ya tenía un precio de $\$ 9460$ ( $\$ 1080$ menos que el 29 de noviembre de 2017). Lo anterior indica dos cosas: por un lado, que la divulgación del rumor afectó más que la divulgación de la verdad; por otro lado, que sigue siendo un enigma el cumplimiento de la hipótesis de mercado eficiente por parte de la acción de Argos, enigma a ser resuelto por los lectores aunque el autor se inclina a su no cumplimiento.

Esta situación del cartel muestra que la información no contable también puede crear una imagen sesgada sobre la empresa, pues tal situación no se la imaginó el valorador unos meses antes durante la valoración cuando los reportes integrados dieron la imagen de que la empresa era un negocio ético en todos sus aspectos. Si el valorador hubiera estado ante una decisión real de inversión, y las noticias de rumor y confirmación se hubieran emitido en la época de valoración de la empresa, quizás el valorador hubiese dudado sobre la compra de la misma. Ahora, en otro escenario hipotético, si el valorador no asumiera el rol de comprador de la empresa sino que como capitalista rentista hubiese invertido fortunas en acciones en la fecha de valoración, legitimado por el conjunto de información, habría sufrido grandes pérdidas en el desplome.

Entonces, es menester cuestionar las pretensiones objetivistas de las ciencias económicas; es necesario mirar los aspectos más allá de los simples datos presentados al tomar una decisión de inversión. Esto porque aquellos datos capturados cuando se valoran empresas son trasladados como supuestos a los modelos de valoración (tal y como ocurrió en la valoración de Argos); y los supuestos de alguna u otra forma terminan "siendo realidad" para quien los hace legítimos vía confianza. De nuevo a 
lo planteado por Théret (2015): se cree que la moneda tiene valor aunque físicamente solamente es papel ${ }^{19}$; esa creencia hace que la aceptación de la moneda como medio de pago sea algo racional, y de esta manera, el hecho de que la moneda tiene valor se vuelve una "realidad aceptada". Así, el papel moneda no tiene valor a la luz de la verdad absoluta, pero como realidad aceptada sí tiene valor porque socialmente se dispuso así. Si se lleva esa situación de la moneda al tema de la valoración de empresas, hay que decir que cuando a partir del conjunto de información se hace una apreciación sobre una empresa, por ejemplo, que esta es muy rentable o ética, a la luz de la verdad absoluta puede no ser rentable ni ética, pero si dicho conjunto indica que sí es rentable y ética, entonces se vuelve una realidad aceptada la apreciación de que "la empresa es rentable y ética".

Por lo anterior, es limitada la racionalidad de los inversionistas en sus decisiones de inversión a partir de la valoración de empresas; pues podrían llegar a confiar sus decisiones en realidades excesivamente artificiales, con consecuencias adversas tanto para ellos como para la sociedad, que no siempre son tan tenues como en el caso de Cementos Argos. El desplome de la acción de Argos fue sin duda una situación negativa; sin embargo, las consecuencias adversas se quedaron solamente en una sanción para la empresa y en probables pérdidas para varios inversionistas reales, pero la empresa sigue en pie, y no se conocen afectaciones relevantes a la sociedad por tal situación. En contraste, en otras situaciones son fuertes los impactos causados por la confianza en la excesiva artificialidad de los datos del conjunto de información, como en el caso de Enron.

En octubre de 2001 la SEC empieza a investigar a la compañía... sale a la luz tanto el engaño de los directivos, como la deuda ocultada con manipulaciones. Por lo anterior, la cotización de Enron se desploma, siendo la quiebra más importante de USA... El asombro es que antes de octubre de 2001

19 Como dice el grupo Neurosis en su canción "Convención ancestral”. Esto, no por promover el grupo o su respectivo género musical (que no es de gusto del autor), sino por llamar la atención sobre las verdades de la letra de la canción. 
Enron daba una imagen de empresa ejemplar. Era vista como un modelo de negocio complejo pero atractivo. (Aglietta y Rebérioux, 2009, p. 285).

Esta situación resulta muy familiar a lo que ocurrió en la valoración de Cementos Argos: en principio el inversionista valorador pasa por una ilusión (la buena imagen de la empresa) y después llega a la desilusión. La diferencia entre ambas situaciones es el impacto: la situación de Argos tuvo consecuencias negativas, pero no tan abismalmente negativas como ha ocurrido en otros casos como el de Enron.

Hasta la fecha (22 de mayo de 2019) los precios de las acciones (ordinaria y preferencial) no recuperan el comportamiento que tuvieron a finales de 2017. Respecto al precio de la acción ordinaria, que es el que más interesa, no ha podido recuperarse desde el lapso de la emisión de las malas noticias. Es innegable que el precio de la acción subió después de la noticia de confirmación de la conducta; no obstante, eso puede interpretarse (en concepto del autor) como un movimiento corrector de tendencia con pico en los últimos días de enero de 2018, para tomar una nueva tendencia a la baja $^{20}$, pues también es claro que desde los últimos días de enero de 2018 el precio de la acción lo que ha hecho las más de las veces es caer y caer, a tal punto de que ha llegado a estar incluso por debajo de $\$ 7000$. El comportamiento del precio de la acción de Argos durante 2018 y lo corrido de 2019 no se compara en nada a lo que fue durante 2017. En ocasiones la acción ha tenido subidas significativas, pero no ha recuperado el comportamiento que tenía en el período octubre 2017-enero 2018.

\section{Conclusiones}

A partir de la valoración de Cementos Argos S. A. se muestra que cuando un inversionista hace valoración de empresas, al utilizar para tal valoración un conjunto de información (contable y no contable) se encuentra con cinco tipos de problemas distintos:

20 También es posible hallar una explicación a esa paradójica subida a partir del no cumplimiento de la hipótesis de mercado eficiente. 
A. Problemas originados por la valoración misma, tales como la escasez de recursos en la valoración (p. ej. tiempo, dinero) y la natural incertidumbre al predecir el futuro tanto de la empresa como de su entorno.

B. Problemas originados por el valorador, tales como la subjetividad, prejuicios y creencias que este tenga en la valoración. En este sentido, la experiencia del valorador en valorar empresas, su conocimiento previo y personalidad resultan factores determinantes porque influyen en últimas en la rigurosidad de la valoración y en los supuestos empleados en la misma.

C. Problemas originados por el conjunto de información, como las metodologías utilizadas para producir la información, la complejidad de dichas metodologías, el modelo contable y la ausencia o suficiencia de información de las fuentes. Incluso, el mismo conjunto de información puede implicar que en la valoración se siga un camino o método específico.

D. Problemas originados por las personas detrás de la construcción del conjunto de información, como el pluralismo y ambigüedad de opiniones sobre una variable específica utilizada en la valoración (p. ej. crecimiento futuro del PIB), los conflictos de intereses de estas personas respecto a la información emitida, el profesionalismo de dichas personas y su subjetividad involucrada en la preparación del conjunto de información y en las decisiones propias que puedan tomar y que afectan la valoración (p. ej. una decisión gerencial).

E. Problemas originados por otros factores, como las características específicas de la industria valorada, la desconfianza que pueda tener la sociedad ante un rumor divulgado y los hechos ocurridos que no están bajo control de la empresa.

Debido a todo este tipo de problemas el inversionista valorador puede tener una imagen sesgada sobre la empresa en valoración y, por ende, tener inconvenientes para establecer adecuadamente tanto si es viable comprar la empresa como el rango de valores (tal como ocurrió con Cementos Argos). Pues todas las falencias del conjunto de información muestran que tal conjunto tiene una confiabilidad limitada; como su confiabilidad es limitada, asimismo la racionalidad del inversionista en la toma de decisiones de inversión es limitada, pues existe el riesgo de que tome decisiones adversas. 
El inversionista debería ser prudente en sus decisiones de inversión en una empresa específica al basarse en la valoración de empresas, pues si le da una confianza absoluta al conjunto de información, puede actuar desenfrenadamente, con consecuencias adversas.

\section{Referencias}

Aglietta M., y Rebérioux A. (eds.) (2009). El capitalismo financiero a la deriva. El debate sobre el gobierno de empresa. Bogotá D. C., Colombia: Editorial Universidad Externado de Colombia.

Argos. (2017). Acción y ADR. Recuperado de https://www.argos.co/ir/ informacion-financiera/accion

Bezemer, D. (2012). Modelos contables y comprensión de la crisis financiera. Revista de economía institucional, 14(26), 47-76.

Comín, F. (2013). La economía de la edad de piedra y la revolución neolítica. Historia económica mundial. De los orígenes a la actualidad. Madrid, España: Alianza Editorial.

El Tiempo. (2017). Sí hubo cartel del cemento en Colombia: Superindustria. 13 de octubre. Recuperado de http://www.eltiempo.com/economia/empresas/ holcim-argos-y-cemex-vinculados-a-cartel-del-cemento-en-colombia-140774

Hidalgo, A. (1978). El principio de racionalidad limitada de H. A. Simon y el Premio Nobel de Economía. El basilisco, septiembre-octubre (4), 68-79. http://www. fgbueno.es/bas/pdf/bas10407.pdf

IASB. (2016). International Accounting Standards Board (Comité de Normas Internacionales de Contabilidad). Norma NIC 29, Información Financiera en Economías Hiperinflacionarias.

Parra, A. (2013). Valoración de empresas: métodos de valoración. Revista Contexto, 2(1) $87-100$.

Robledo, P. (2017). La lucha de la SIC contra la corrupción. La Nación, 4 de noviembre. http://www.lanacion.com.co/2017/11/04/la-lucha-la-sic-la-corrupcion/

Santandreu, P., y Torres, J. (2012). Selección del método de valoración de empresas en función de la empresa y el ciclo económico: El modelo QQC. Revista de Contabilidad y Dirección. 15, 115-132. Recuperado de http://www.accid. 
org/revista/documents/Seleccion_del_metodo_de_valoracion_de_empresas_en_funcion_.pdf

Sarmiento, J., y Cayón, E. (2003). Modelos de valoración de empresas. Recuperado de http://www.javeriana.edu.co/decisiones/Julio/valoracion.pdf

Stiglitz, J. E. (2003). Los felices 90: la semilla de la destrucción. Madrid, España: Taurus.

Superintendencia de Industria y Comercio. (2017). Por cartelización empresarial en el mercado del cemento, Superindustria sanciona a ARGOS, CEMEX y HOLCIM. Recuperado de http://www.sic.gov.co/noticias/por-cartelizacionempresarial-en-el-mercado-del-cemento-superindustria-sanciona-a-argoscemex-y-holcim

Théret, B. (2015). El trípode de la moneda: deuda, soberanía y confianza. En A. Wilkis y A. Roig (eds.), El laberinto de la moneda y las finanzas. (pp. 67-83). Buenos Aires, Argentina: Biblos. 